\title{
LES FLUCTUATIONS À COURT TERME DU CLIMAT ET L'INTERPRÉTATION DES OBSERVATIONS RÉCENTES EN TERMES D'EFFET DE SERRE
}

\author{
Jean-Claude André(1) et Jean-François Royer ${ }^{(2)}$ \\ (1) Centre européen de recherche et de formation avancée \\ en calcul scientifique (Cerfacs) \\ 42, avenue Gaspard-Coriolis \\ 31057 Toulouse Cedex 1 \\ (2) Météo-France \\ Centre national de recherches météorologiques (CNRM) \\ Toulouse
}

Ndlr : Cet article a été publié initialement dans les Comptes rendus de l'Académie des sciences, série II, tome 328, $\mathrm{n}^{\circ}$ 4, fascicule a, février 1999, p. 261-272. Nous remercions les Comptes rendus de l'Académie des sciences de nous avoir autorisés à le publier dans La Météorologie.

RÉSUMÉ

Les simulations du climat futur, réalisées avec des modèles couplés de la circulation générale de l'atmosphère et de l'océan, prévoient que l'augmentation de la concentration des gaz à effet de serre rejetés dans l'atmosphère par l'activité de l'homme devrait avoir un effet important sur le climat du prochain siècle. La mise en évidence de l'impact climatique produit par l'augmentation rapide de la concentration du gaz carbonique atmosphérique au cours des dernières décennies est rendue difficile par la forte variabilité interannuelle du climat, et nécessite l'utilisation de méthodes statistiques combinant plusieurs indicateurs climatiques (méthode des «empreintes » climatiques) pour améliorer la détection d'une perturbation anthropique. Dans cet article, on passe en revue l'évolution au cours des dernières décennies de plusieurs indicateurs climatiques montrant le réchauffement global, sa répartition géographique, le niveau de l'océan, le cycle hydrologique et les réponses de la végétation, en les comparant aux résultats des scénarios climatiques prédits par les modèles. La cohérence entre les résultats des modèles et les tendances climatiques observées indique que l'effet de serre additionnel commence à devenir détectable dans les séries climatiques récentes.

\footnotetext{
ABSTRACT Short-term climatic fluctuations and the interpretation of recent observations in terms of greenhouse effect

Simulations of future climate, made with coupled general circulation models of the atmosphere and ocean, predict that the increase of the concentration of greenhouse gases released in the atmosphere by man's activities will have a large influence on the climate of the next century. The identification of the climatic impact produced by the rapid increase in carbon dioxide concentration in recent decades is made difficult by the strong interannual climate variability. Statistical techniques are required to combine several climatic indicators (method of climatic "fingerprints") to improve the detection of a possible anthropogenic perturbation. In this paper we review the evolution through the last decades of several climate indicators showing global warming, its geographical distribution, sea level, the hydrological cycle and the response of vegetation, and we compare them to the model results predicted in climate scenarios. The coherence between model results and observed climatic trends shows that the additional greenhouse effect is starting to become detectable in recent climatic data.
} 
INTRODUCTION

\section{DES PRÉDICTIONS DES MODĖLES CLIMATIQUES AUX OBSERVATIONS Le réchauffement global}

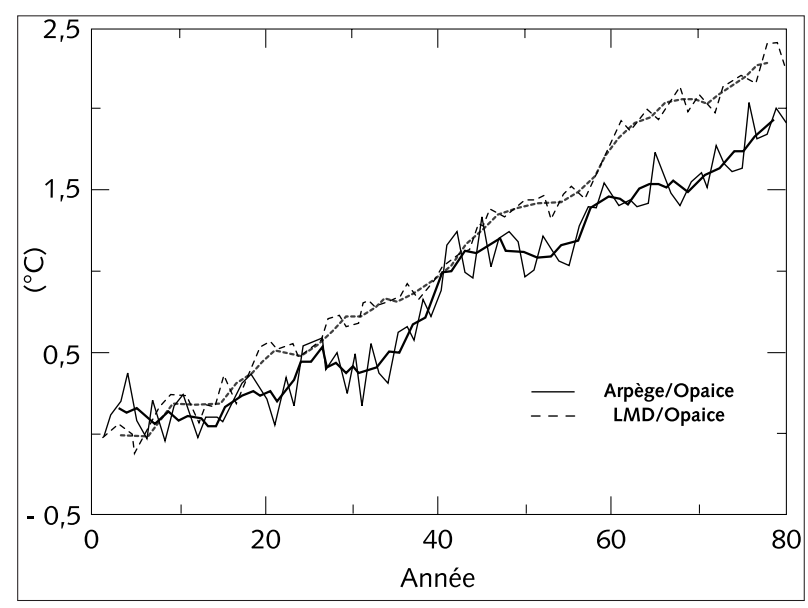

Figure 1 - Évolution, pour les 80 prochaines années, de la moyenne globale de la température de l'air à $2 \mathrm{~m}$, en réponse à un accroissement graduel de $1 \%$ par an de la concentration du $\mathrm{CO}_{2}$ atmosphérique, calculée selon deux modèles couplés : Arpège/ Opaice (trait plein) et LMD/Opaice (tireté). Les valeurs annuelles sont représentées en traits fins, et les moyennes glissantes sur cinq ans en traits gras. (D'après Barthelet et al., 1998)
Le climat terrestre fluctue sur une très large gamme d'échelles temporelles, depuis les fluctuations à plus court terme, qui concernent la variabilité mensuelle et saisonnière, et les fluctuations interannuelles, dont l'exemple le plus connu est celui de l'Oscillation australe et du phénomène El Niño (Enso), résultant de l'interaction entre l'atmosphère et l'océan superficiel tropical (Philander, 1990), jusqu'aux fluctuations décennales et centenaires, mettant en jeu les interactions entre l'atmosphère, l'hydrologie continentale et l'océan profond, ainsi qu'aux fluctuations à l'échelle de plusieurs dizaines de millénaires, telles que la succession des périodes glaciaires et interglaciaires liées aux perturbations astronomiques de l'orbite terrestre - théorie de Milankovitch (Berger, 1988).

L'homme est, par ailleurs, en train de modifier globalement les propriétés chimiques de l'atmosphère, en y injectant des quantités croissantes de gaz radiativement actifs (les gaz à effet de serre, GES) produites par ses activités industrielles ou agricoles. Les conséquences prévisibles de cette évolution de la composition chimique de l'atmosphère conduisent à un réchauffement du climat, et particulièrement de la basse atmosphère, sur une échelle de temps se mesurant en décennies.

La question abordée dans cette note de synthèse concerne la possible détection et l'identification d'un « signal » climatique résultant de l'action anthropique, au sein $\mathrm{du}$ « bruit » issu de la variabilité naturelle du climat. Pour ce faire, un certain nombre d'observations climatiques sont passées en revue et discutées en termes d'appartenance possible au signal anthropique.

Aucune de ces observations n'étant encore suffisante en elle-même pour permettre de trancher indubitablement la question, il est nécessaire, pour dégager la meilleure réponse possible, non seulement de recourir au faisceau complet que ces observations représentent, mais aussi de les discuter par rapport aux prédictions de la réponse du système climatique à l'augmentation de la quantité des GES qui sont fournies par les modèles couplés océan-atmosphère.

Six paramètres climatiques sont successivement examinés et présentés par ordre décroissant quant à leur signification vis-à-vis de la mise en évidence du signal anthropique de réchauffement dû à l'augmentation de l'effet de serre.

Les modèles climatiques indiquent, depuis maintenant près de vingt ans, que sous l'action d'une augmentation de la quantité des GES, la température moyenne à la surface de notre planète doit augmenter. Les chiffres de réchauffement moyen global les plus généralement cités sont compris dans une fourchette comprise entre $1,5 \mathrm{~K}$ et 3,5 K pour la température à l'équilibre correspondant à un doublement de la concentration du gaz carbonique atmosphérique $\left(\mathrm{CO}_{2}\right)$ par rapport à sa valeur actuelle. Dans le cas d'une augmentation graduelle du $\mathrm{CO}_{2}$, le réchauffement obtenu pourrait être un peu plus faible au moment du doublement, par suite de l'inertie thermique des océans qui peut retarder d'une dizaine d'années leur mise en équilibre. À titre d'exemple, la figure 1 montre les résultats obtenus avec deux modèles couplés océan-atmosphère constitués du même modèle océanique, mais de modèles atmosphériques différents (Barthelet et al., 1998). À l'horizon de soixante-dix ans, date du doublement de la quantité de $\mathrm{CO}_{2}$ dans ces simulations où le taux atmosphérique de $\mathrm{CO}_{2}$ est supposé augmenter de $1 \%$ par an, l'un des modèles indique un réchauffement de 1,6 K, tandis que l'autre conduit à un réchauffement de $2 \mathrm{~K}$. Il est à signaler ici que les deux modèles présentés ne prennent pas spécifiquement en compte l'effet modérateur d'une augmentation concomitante possible de la quantité d'aérosols. Toutefois, les modélisations climatiques prenant en compte ces effets conduisent aussi à prédire un réchauffement global, quoique d'amplitude un peu moins importante (Mitchell et al., 1995 ; Manabe et Stouffer, 1997 ; Hansen et al., 1997 ; Carnell et Senior, 1998 ; Reader et Boer, 1998). 
Figure 2 - Évolution de la température moyenne de l'air pendant la période 18601997, calculée à partir des observations terrestres et maritimes (Jones, 1994 ; Parker et al., 1994). Les triangles représentent les moyennes des hémisphères nord (pointe en haut) et sud (pointe en bas), la courbe en trait plein une moyenne glissante sur cinq ans. (Données de P. Jones, disponibles au Climatic Research Unit,

http://www.cru.uea.ac.uk/cru/data/temperat.htm)

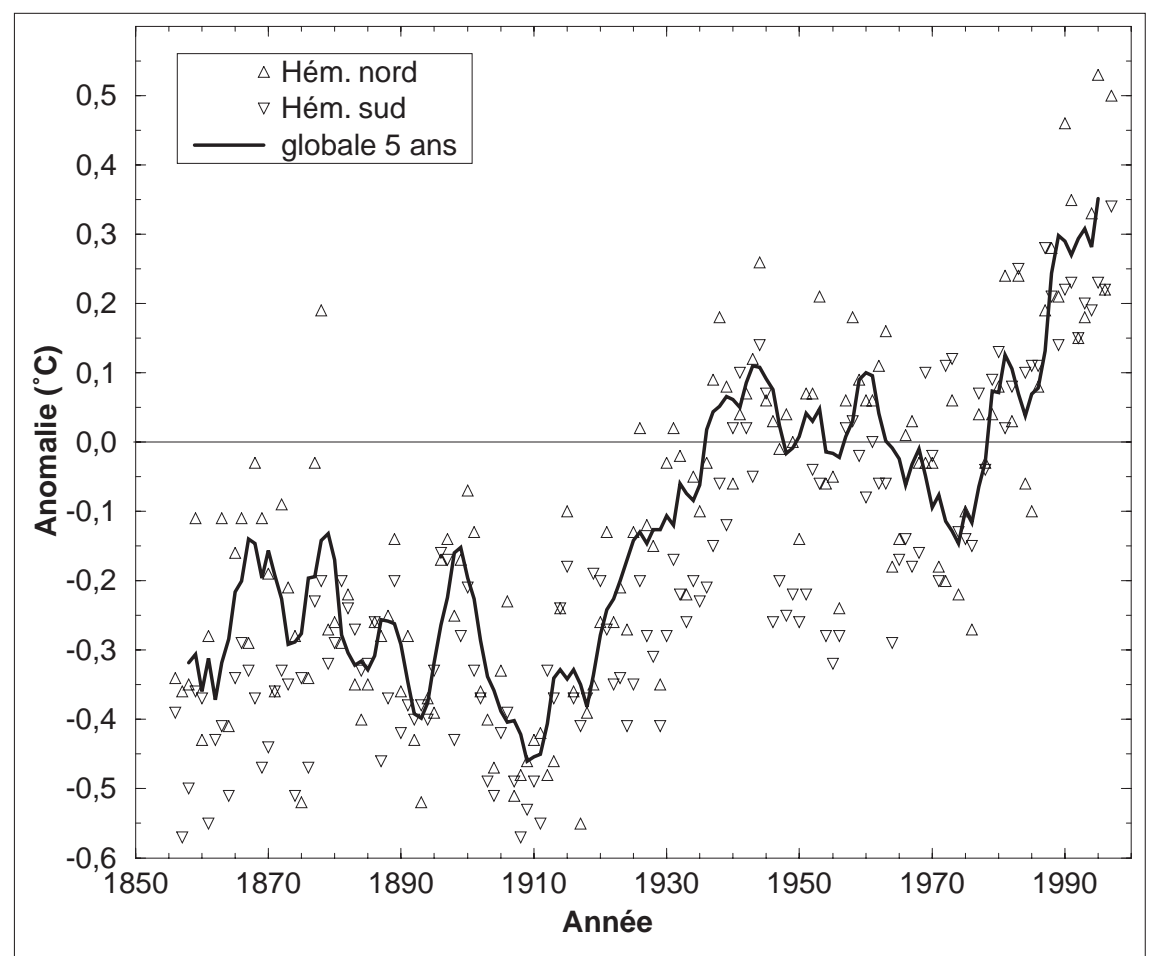

L'évolution de la température atmosphérique globale moyenne observée depuis environ un siècle est représentée sur la figure 2. On y constate deux éléments particulièrement notables. D'une part, la croissance de la température globale depuis le début du siècle a été de l'ordre de 0,6 K (avec des paliers et des accélérations non sans similitudes avec la courbe la plus basse de la figure 1). D'autre part, la décennie la plus récente est aussi la plus chaude jamais enregistrée depuis qu'existent les réseaux météorologiques. Ces deux éléments sont ceux les plus couramment invoqués (Houghton et al., 1996) pour confirmer le début d'apparition de la réponse climatique à l'effet de serre additionnel.

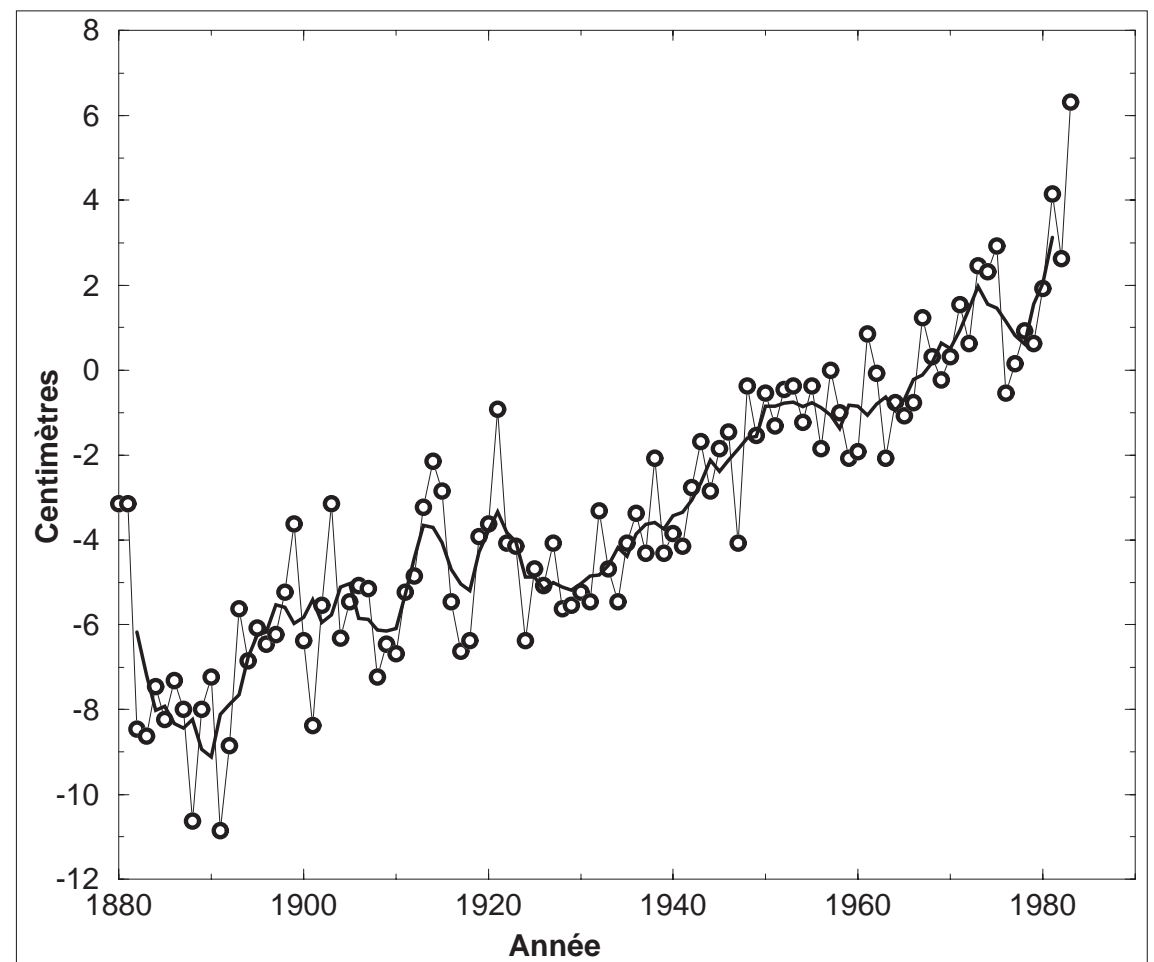

Figure 3 - Évolution du niveau moyen de la mer (en $\mathrm{cm}$ ) au cours des cent dernières années, par rapport au niveau moyen pour la période 1951-1970. Les cercles reliés par une ligne en trait fin représentent les valeurs annuelles, la courbe en trait épais leur moyenne mobile sur cinq ans. (D'après Gornitz, 1995)

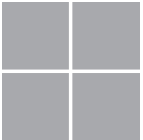


L'élévation du niveau de la mer

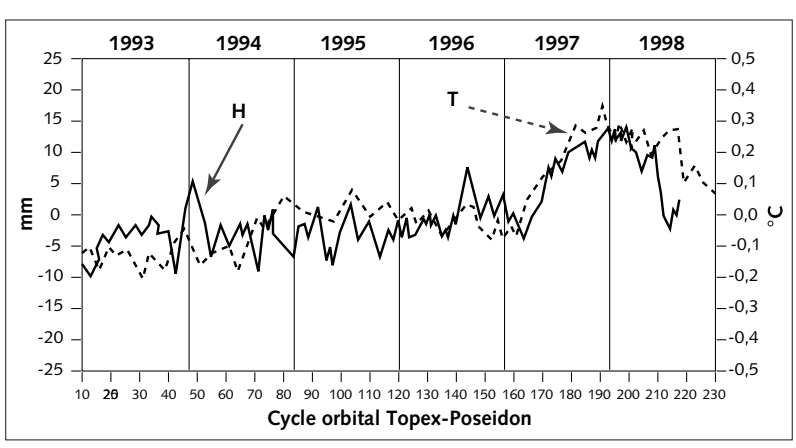

Figure 4 - Variations du niveau moyen de la mer $(\mathrm{H}$, en $\mathrm{mm})$ et de la température de surface d'océan $\left(T\right.$, en ${ }^{\circ} \mathrm{C}$ ) pendant la période décembre 1992 à mai 1998, selon les mesures de TopexPoseidon. (D'après Cazenave et al., 1998)
Si l'estimation de la température moyenne de la surface de la planète n'est pas un exercice sans difficulté (Jones, 1995 ; Basist et Chelliah, 1997), il existe un effet assez directement lié à ces aspects thermiques, mais dont l'estimation ne pose pas les mêmes problèmes : la variation du niveau moyen des océans (Le Provost, 1991 ; Pirazzoli, 1996). Ces variations sont dues à plusieurs facteurs, tels que les variations isostatiques, les rebonds glaciaires, la variation du volume des glaces continentales, et, principalement, à l'effet de dilatation de l'eau de mer, ce dernier représentant actuellement environ la moitié du signal total. Les mesures sur la durée du siècle passé qui sont présentées sur la figure 3 confirment qu'une élévation continue est en cours, avec une amplitude de l'ordre de $10 \mathrm{~cm}$ par siècle (Gornitz, 1995). Cet ordre de grandeur, mesuré à partir de réseaux in situ, est confirmé directement à l'échelle globale par les mesures du satellite altimétrique Topex-Poseidon : l'analyse des données reçues depuis la mise en orbite du satellite indique, en effet, un taux moyen annuel d'élévation du niveau de la mer de $1 \mathrm{~mm}$ par an, cf. figure 4 (Cazenave et al., 1998).

\section{La distribution spatiale du réchauffement}

Figure 5 - Répartition géographique des tendances des températures annuelles (en degrés par siècle), calculées sur la période 1891-1990 à partir des données d'observation sur une grille de $5^{\circ} \times 5^{\circ}$ (Jones, 1994) disponibles au Climate Research Unit (http://www.cru.uea.ac.uk/cru/data/temperat.htm).
De façon probablement plus convaincante, il faut souligner que, non seulement la température moyenne de la planète augmente actuellement à un rythme compatible avec celui prédit par les modèles en réponse à l'effet de serre additionnel, mais aussi que la distribution géographique du réchauffement observé, avec des régions de haute latitude beaucoup plus affectées pendant l'hiver correspondant (figure 5), est tout à fait semblable à celle issue des prédictions par modélisation (figure 6, Barthelet et al., 1998).

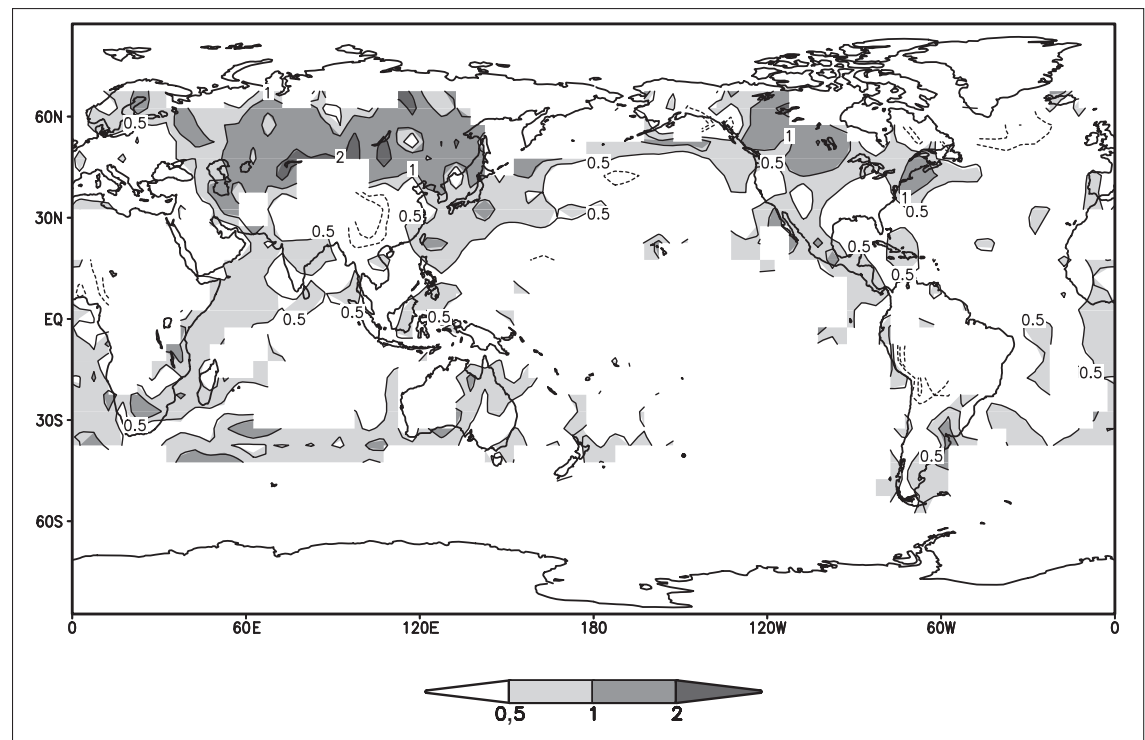

Figure 6 - Répartition géographique du réchauffement en surface pour la période de doublement de la concentration du $\mathrm{CO}_{2}$ atmosphérique, calculée par deux modèles couplés selon le scénario de la figure 1 . (D'après Barthelet et al., 1998)

\section{LMD/Opaice}

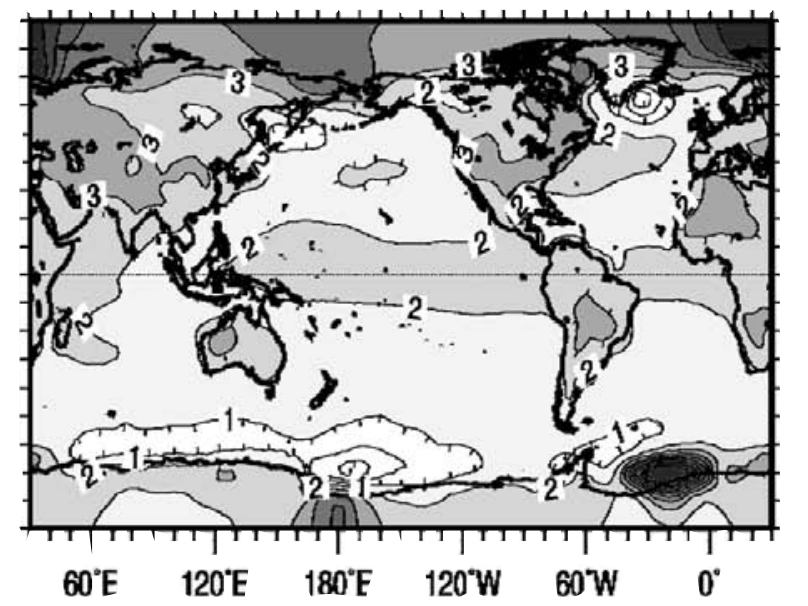


Cette ressemblance peut être formalisée par le calcul d'un coefficient de corrélation entre les cartes de la répartition spatiale des tendances climatiques observées et les cartes de la réponse à l'augmentation de l'effet de serre prévue par le modèle, puis testée par des méthodes statistiques rigoureuses (Hegerl et al., 1996). Cette méthode, qui consiste à comparer la forme du signal climatique observé à des réponses types produites par divers forçages, est connue sous le nom de méthode des « empreintes climatiques »(fingerprints). Cela peut permettre de reconnaître et de séparer les réponses climatiques provenant de différents forçages tels que l'insolation, le gaz carbonique, les éruptions volcaniques, les aérosols d'origine anthropique, à condition d'avoir pu déterminer au préalable leurs réponses caractéristiques à l'aide d'un modèle adéquat. Par exemple, une augmentation de l'insolation produit un réchauffement troposphérique assez semblable à celui des GES, mais ne produit pas le refroidissement stratosphérique caractéristique lié à l'effet de serre, tandis que le forçage par les aérosols est limité au voisinage des sources sur les continents de l'hémisphère nord. On voit donc que la combinaison de plusieurs types de données climatiques, en surface et en altitude, peut fournir des indices très précieux sur les causes des fluctuations climatiques. Cette méthode des empreintes a été mise en œuvre récemment dans plusieurs études (Santer et al., 1995 ; Tett et al., 1996), qui ont conclu que les tendances observées au cours des dernières décennies pouvaient être attribuées en grande partie aux activités humaines, notamment à l'action combinée de l'effet global du gaz carbonique et de l'effet local des aérosols contenant du soufre.

\section{L'augmentation de croissance de la végétation}

De simples considérations qualitatives sur la physiologie des plantes indiquent qu'en présence simultanée d'un accroissement de la quantité de gaz carbonique et d'une élévation de la température, si celle-ci est suboptimale, comme c'est généralement le cas aux moyennes et hautes latitudes, et à la condition que la ressource en eau ne devienne pas un facteur limitant, la photosynthèse et la production de biomasse doivent augmenter (Cure et Acock, 1986 ; Allen et al., 1996), fait confirmé quantitativement par des études en chambre-laboratoire (Casella et al., 1996 ; Mousseau et al., 1996 ; Saugier, 1998).

L'augmentation de l'amplitude du cycle annuel du $\mathrm{CO}_{2}$ atmosphérique, observée depuis 1960 (20 \% à Mauna Loa et près du double autour de l'Arctique), indique une assimilation accrue du $\mathrm{CO}_{2}$ par la végétation, qui ne peut être interprétée que par un allongement de la saison de croissance, en réponse à un réchauffement des hautes latitudes de l'hémisphère nord en hiver et au printemps, la stimulation de la photosynthèse par l'augmentation concomitante du $\mathrm{CO}_{2}$ ne pouvant expliquer par elle-même que moins de $10 \%$ du signal (Keeling et al., 1996). Ce résultat a été confirmé par des observations par satellite des indices de végétation (NDVI),

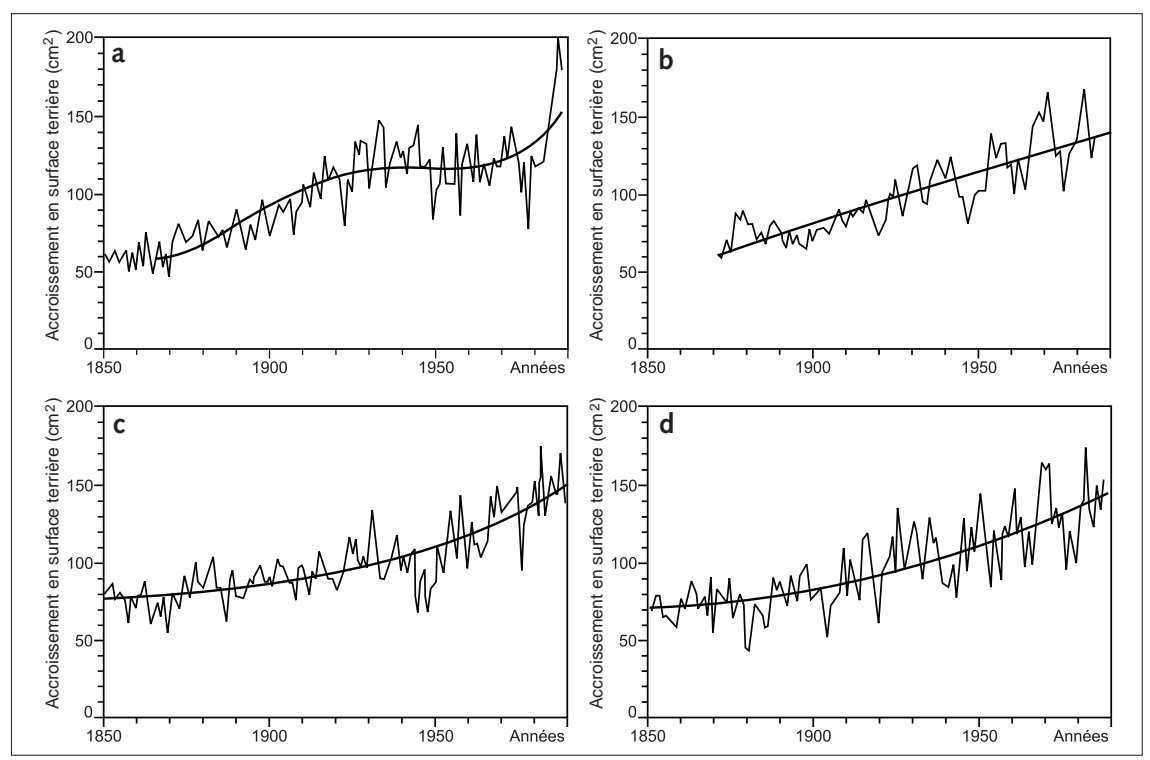

Figure 7 - Augmentation depuis 1850 de la croissance radiale (mesurée en $\mathrm{cm}^{2}$ de surface terrière) de quatre essences forestières de l'Est de la France (d'après Becker et al., 1995) : (a) du sapin, (b) de l'épicéa, (c) du hêtre, dans les Vosges, et (d) du chêne sessile, sur le plateau lorrain. 

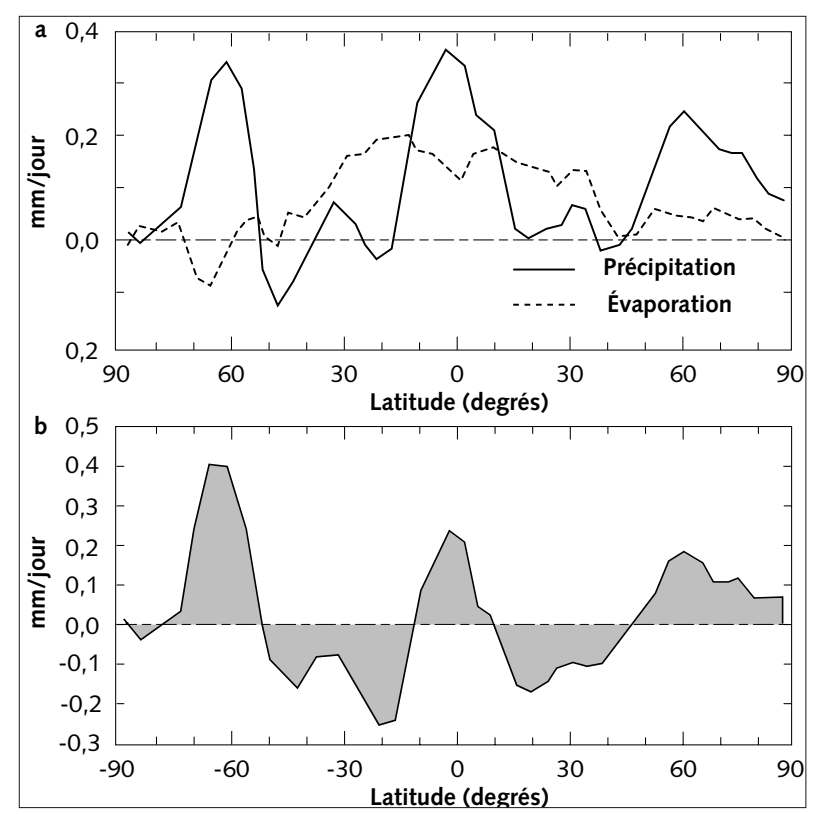

Figure 8 - Réponse du taux de précipitation $P$ et d'évaporation $E$ (a), et de leur différence représentant le bilan hydrique en surface P-E (b), en mm par jour, calculés par un modèle couplé, pour la période de doublement du $\mathrm{CO}_{2}$ atmosphérique dans le scénario de la figure 1. (Barthelet et al., 1998)

indiquant une augmentation de la photosynthèse et un allongement de douze jours de la saison de croissance active entre 45 et $70^{\circ} \mathrm{N}$ au cours de la décennie 1981-1991 (Myneni et al., 1997). Un inventaire des ressources forestières européennes a montré que leur biomasse a effectivement augmenté de 1971 à 1990 (Kauppi et al., 1992). Il existe malheureusement très peu de mesures de la biomasse à l'échelle globale sur des durées suffisamment longues pour pouvoir être interprétées par rapport au climat de la totalité du siècle écoulé. Toutefois, les mesures de croissance radiale, opérées par les forestiers de l'Inra sur quelques espèces lorraines et vosgiennes, montrent une augmentation indubitable depuis 1850, cf. figure 7 (Becker et al., 1995).

\section{L'accélération du cycle hydrologique}

Au-delà d'une augmentation de la température et des effets qui lui sont thermiquement et directement liés, une question cruciale concerne l'existence de possibles altérations du cycle hydrologique global, principalement à travers son maillon atmosphérique : évaporation, transport vertical et horizontal, précipitation, ruissellement des eaux de surface (Chahine, 1992). Bien que ces paramètres climatiques soient parmi les plus difficiles à estimer et à modéliser, quelques indications semblent confirmer l'existence d'une concomitance entre réchauffement et perturbations du cycle hydrologique de la planète, avec notamment une augmentation de l'intensité et de la fréquence des fortes précipitations (Karl et Knight, 1998).

La figure 8, tirée de Barthelet et al. (1998), indique que, dans une atmosphère enrichie en gaz carbonique et subissant un effet de serre additionnel, l'intensité du cycle hydrologique global augmente d'environ $3 \%$ (figure 8a), l'intensification étant particulièrement sensible dans les régions équatoriales et dans celles de moyennes et hautes latitudes et étant accompagnée d'une stagnation, voire d'un

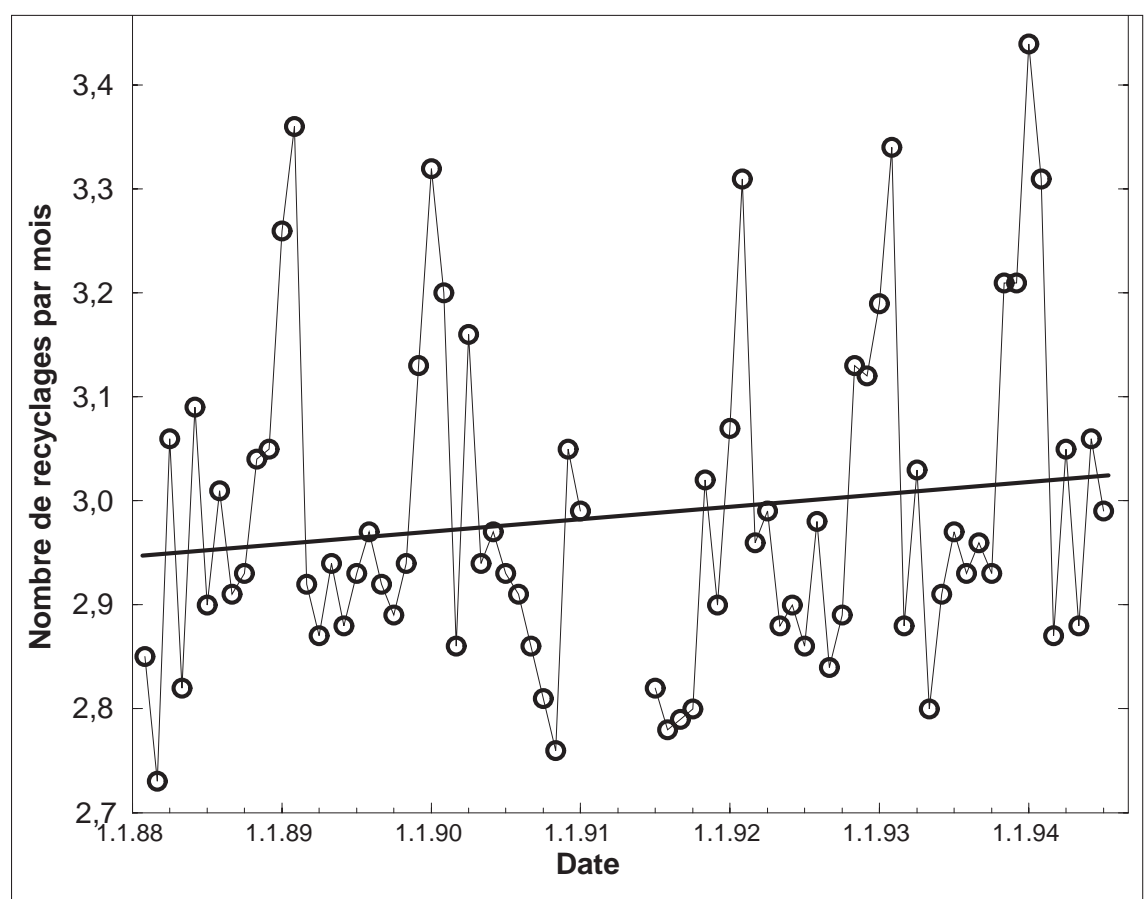

Figure 9 - Variations mensuelles et tendance du taux de recyclage (en nombre de recyclages par mois) de la vapeur d'eau précipitable entre $60^{\circ} \mathrm{S}$ et $60^{\circ} \mathrm{N}$ pour la période 1988-1994. L'accroissement annuel moyen pour la période 1988-1994 est de 0,4 \pm 0,5 \%. (D'après Chahine et al., 1997) 


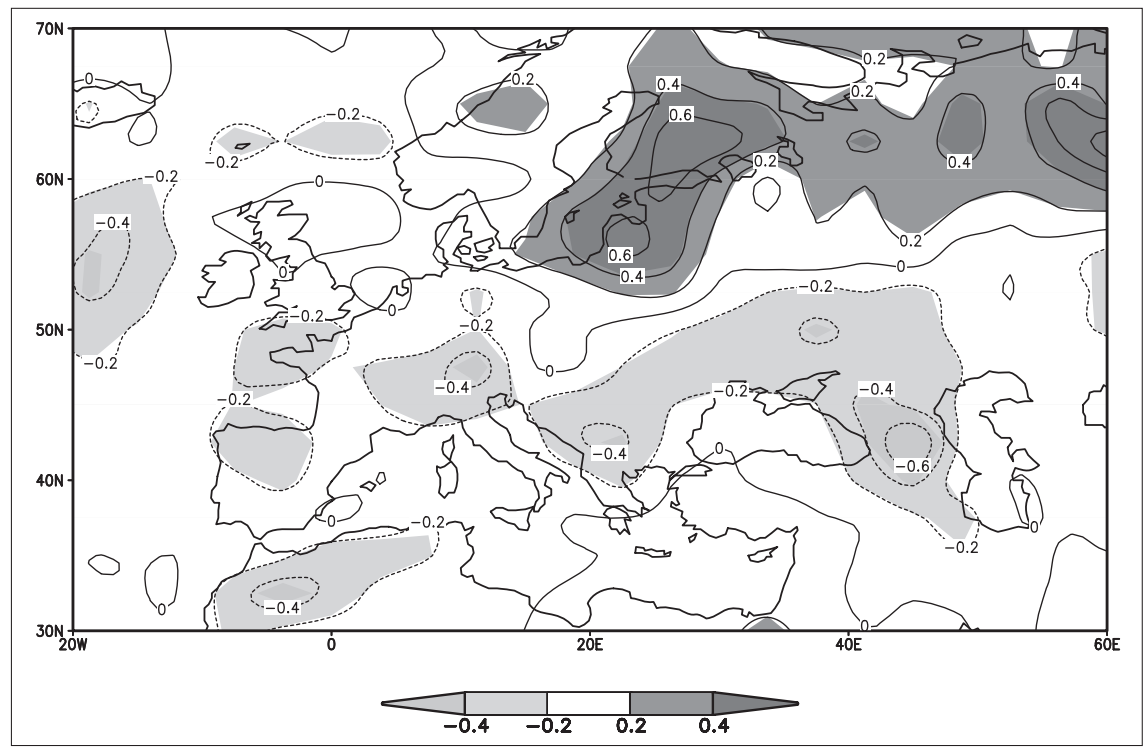

Figure 10 - Réponse à un doublement du $\mathrm{CO}_{2}$ atmosphérique du taux de précipitation sur l'Europe (en $\mathrm{mm}$ par jour) en été boréal (juin-juillet-août) calculé par le modèle de climat de Météo-France avec une résolution accrue sur l'Europe. (D'après Déqué et al., 1998)

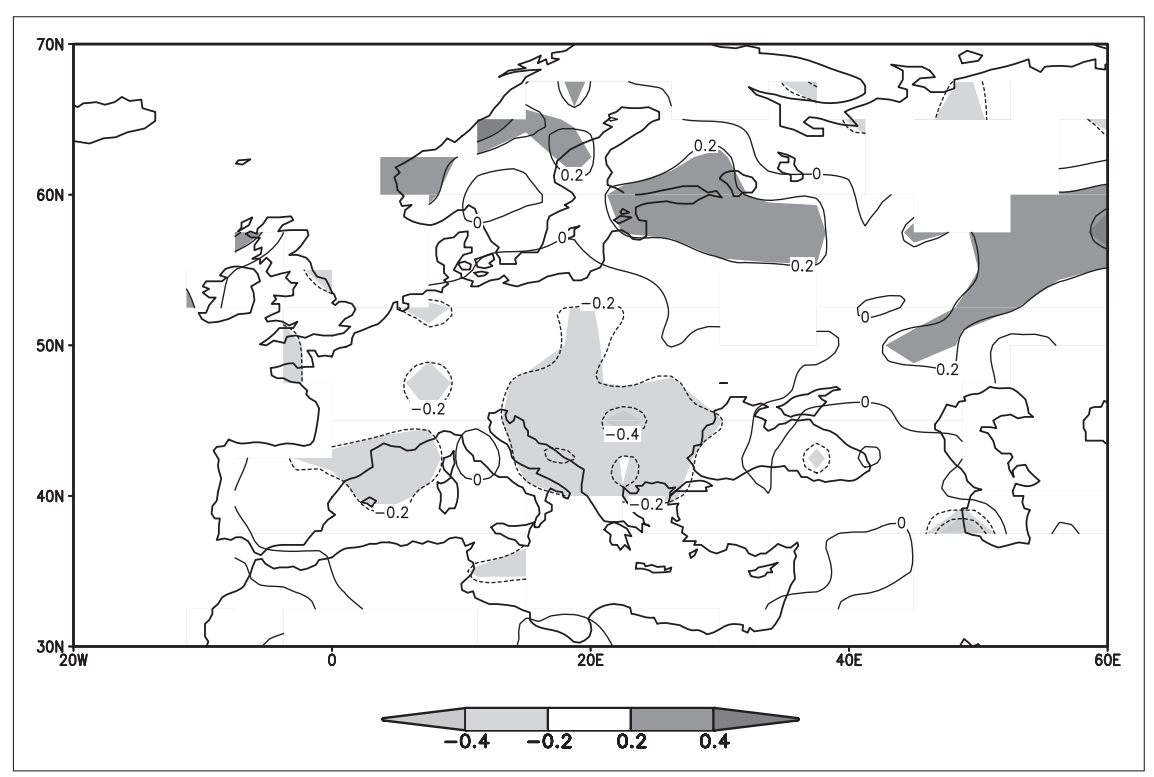

Figure 11 - Anomalies de précipitation sur l'Europe (en mm par jour) pendant l'été boréal de la période 1981-1995 par rapport à la période de référence 1951-1980 (calculées à partir d'observtions

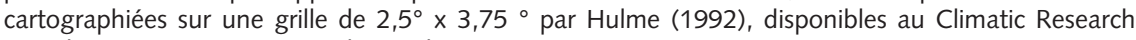
Unit, http://www.cru.uea.ac.uk/cru/data).

\section{Les variations régionales des précipitations}

ralentissement (marqué par un excès de l'évaporation sur les précipitations) dans les régions entre $20^{\circ}$ et $45^{\circ}$ de latitude (figure $8 \mathrm{~b}$ ). Les rares mesures relatives à ces paramètres et disponibles à l'échelle globale paraissent cohérentes avec ces prédictions (figure 9, Chahine et al., 1997) et montrent une tendance à l'augmentation du taux de recyclage de l'eau précipitante. Ce taux de recyclage est défini comme le rapport du taux de précipitation mensuel et du contenu moyen en eau précipitable de l'atmosphère pendant la même période ; il correspond à l'inverse du temps de résidence moyen de la vapeur d'eau atmosphérique, qui est de l'ordre d'une dizaine de jours.

Tout comme dans le cas de la température, l'examen des distributions géographiques des variations peut se révéler au moins aussi informatif que l'examen des valeurs moyennes globales. La figure 10 (Déqué et al., 1998) indique qu'un modèle d'atmosphère soumis à un doublement de la quantité de gaz carbonique prédit, en accord avec les résultats présentés plus haut, une diminution des 
précipitations autour du bassin méditerranéen pendant l'été boréal. Les mesures traitées par Hulme (1992) montrent un effet semblable sur les quinze dernières années (figure 11) et sont compatibles avec le dipôle « assèchement méditerranéen »-« humidification sur l'Europe de l'Ouest et du Nord » visible sur la figure 10 .

Il faut bien sûr souligner ici l'extrême variabilité des précipitations et la difficulté à interpréter une variation mesurée en une partie du globe comme le signe, à elle seule, de l'effet de serre additionnel. Toutefois, lorsqu'elles se manifestent en conjonction avec un ensemble d'autres facteurs, comme on le montre ici, ces variations doivent être prises en considération, ne serait-ce que pour en assurer la surveillance à long terme, seule susceptible de permettre une interprétation pleinement convaincante.

ÉVOLUTION ANTHROPIQUE, FLUCTUATIONS NATURELLES ET BILAN

Un ensemble d'indicateurs convergents vient d'être présenté, qui montre que les effets du réchauffement lié à l'effet de serre additionnel ont très probablement commencé à se manifester. Ce signal lié à la perturbation anthropique est bien sûr encore du même ordre de grandeur que les fluctuations climatiques considérées comme naturelles, parmi lesquelles les grands épisodes de l'Oscillation australe ou Enso (Philander, 1990 ; Battisti et Sarachik, 1995) sont les plus connus (figure 12).

Deux remarques doivent être faites à ce stade.

Il est tout d'abord habituel, et justifié sur le principe, d'attirer l'attention sur le caractère encore incomplet, du point de vue de la physique et de la complexité des phénomènes pris en compte, des modèles climatiques, dont on a vu ici à quel point ils sont consubstantiels à l'étude et à la mise en évidence du réchauffement global. Or, ces modèles sont fondamentalement les mêmes que ceux utilisés pour faire la prévision météorologique opérationnelle et qui sont donc, à ce titre, validés quotidiennement, plus qu'aucun autre modèle de géophysique. Qui plus est, ces modèles sont aussi ceux qui sont mis en œuvre pour prédire les fluctuations à court terme du climat, comme les épisodes Enso. La figure 13 permet de juger directement et quantitativement de la qualité de tels modèles, évaluée par leur capacité à prédire tant l'apparition que l'intensité et la disparition de l'épisode exceptionnel intervenu en 1997-1998.

Il est ensuite intéressant de souligner que Trenberth et Hoar (1997) ont confirmé, par une analyse statistique, une augmentation de la fréquence des épisodes El Niño depuis 1976 (figure 14). Un certain nombre de simulations couplées récentes montrent une telle tendance à l'augmentation de la fréquence des épisodes El Niño accompagnant le réchauffement global (Meehl et Washington, 1996 ; Knutson et al., 1997). Les épisodes El Niño pourraient alors être considérés comme une autre manifestation du surplus d'énergie parvenant au sein du système climatique, qui serait ainsi partiellement évacué par des bouffées plus fortes et plus fréquentes.

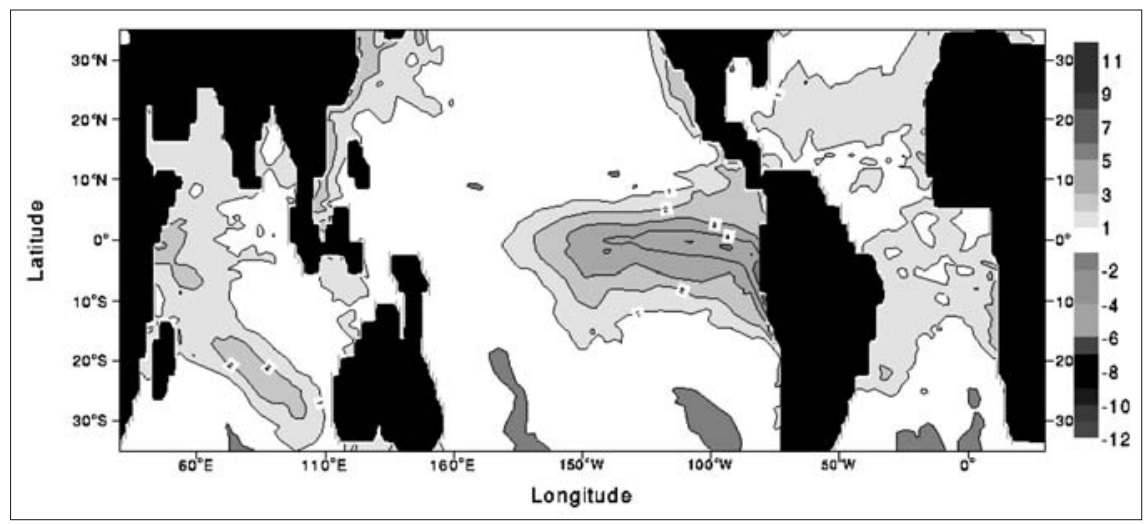

Figure 12 - Anomalie des températures de l'océan à $10 \mathrm{~m}$ de profondeur au cours d'un épisode El Niño (anomalie moyennée sur sept jours autour du 18 janvier 1998), selon les analyses du Centre européen pour les prévisions à moyen terme (CEPMMT, Reading), disponibles sur la page du ECMWF's Seasonal Forecast Project (http://www.ecmwf.int/html/seasonal/index.html). Les isolignes représentent la température potentielle tracée de ${ }^{\circ} \mathrm{C}$ en ${ }^{\circ} \mathrm{C}$. 


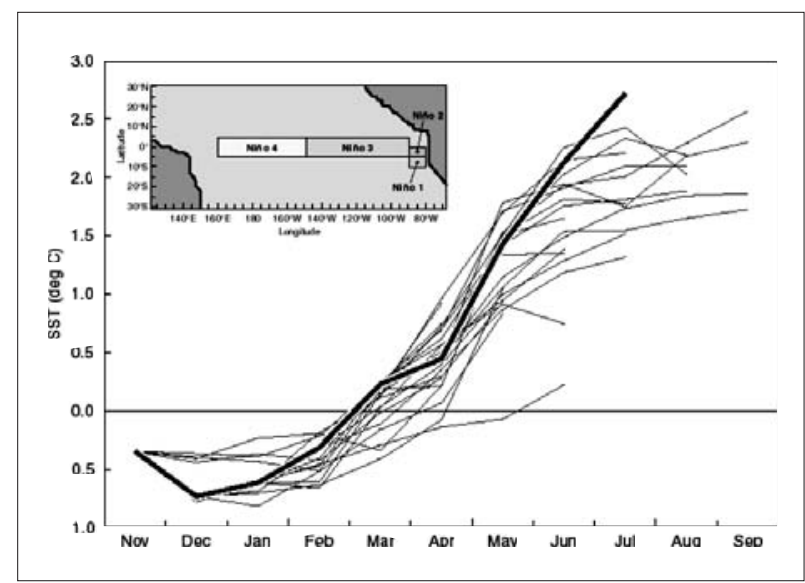

Figure 13 - Prévision saisonnière expérimentale de l'évolution de I'anomalie des températures en surface dans le Pacifique équatorial (région Niño-3), réalisée avec un modèle couplé océan-atmosphère au CEPMMT. La courbe en gras représente l'évolution observée, les courbes en trait fin un ensemble de prévisions décalées dans le temps (disponibles à :

http://www.ecmwf.int/html/seasonal/forecast/index.html).
Le Groupe intergouvernemental d'experts sur l'évolution du climat (GIEC/IPCC) exprime maintenant clairement le bilan de l'ensemble des éléments disponibles quant à la mise en évidence des premières manifestations de l'effet de serre additionnel. Pour reprendre les têtes de chapitre de son rapport de 1995 (Houghton et al., 1996) :

- Greenhouse gas concentrations have continued to increase... (Les concentrations des gaz à effet de serre ont continué à croître...) ;

- Anthropogenic aerosols tend to produce negative radiative forcings... (Les aérosols d'origine anthropique tendent à produire des forçages radiatifs négatifs...);

- Climate has changed over the past century... (Le climat a changé au cours du siècle passé...) ;

- The balance of evidence suggests a discernable human influence on global climate... (Le bilan des preuves suggère une influence humaine discernable sur le climat global...);

- Climate is expected to continue to change in the future... (On peut s'attendre à ce que le climat continue à changer dans l'avenir...) ;

- There are still many uncertainties. (Il y a encore de nombreuses incertitudes).

Il faut souligner, en terminant, que l'existence de ces incertitudes n'entraîne pas que l'hypothèse qualitative de l'existence du réchauffement global soit remise en cause. À cet égard, il n'est pas sans intérêt de remarquer que, au moins à la connaissance des auteurs, aucune étude scientifiquement documentée n'a été publiée - dans les journaux et revues à comité de lecture - qui conduise à des conclusions qualitativement différentes. Quelques chercheurs ont évoqué la possibilité de mécanismes, par exemple une déshydratation de la haute troposphère (Lindzen, 1990), qui seraient susceptibles de compenser en partie le réchauffement directement et radiativement lié à l'accroissement de la quantité de gaz carbonique. De même, Idso (1998), qui exprime depuis longtemps son

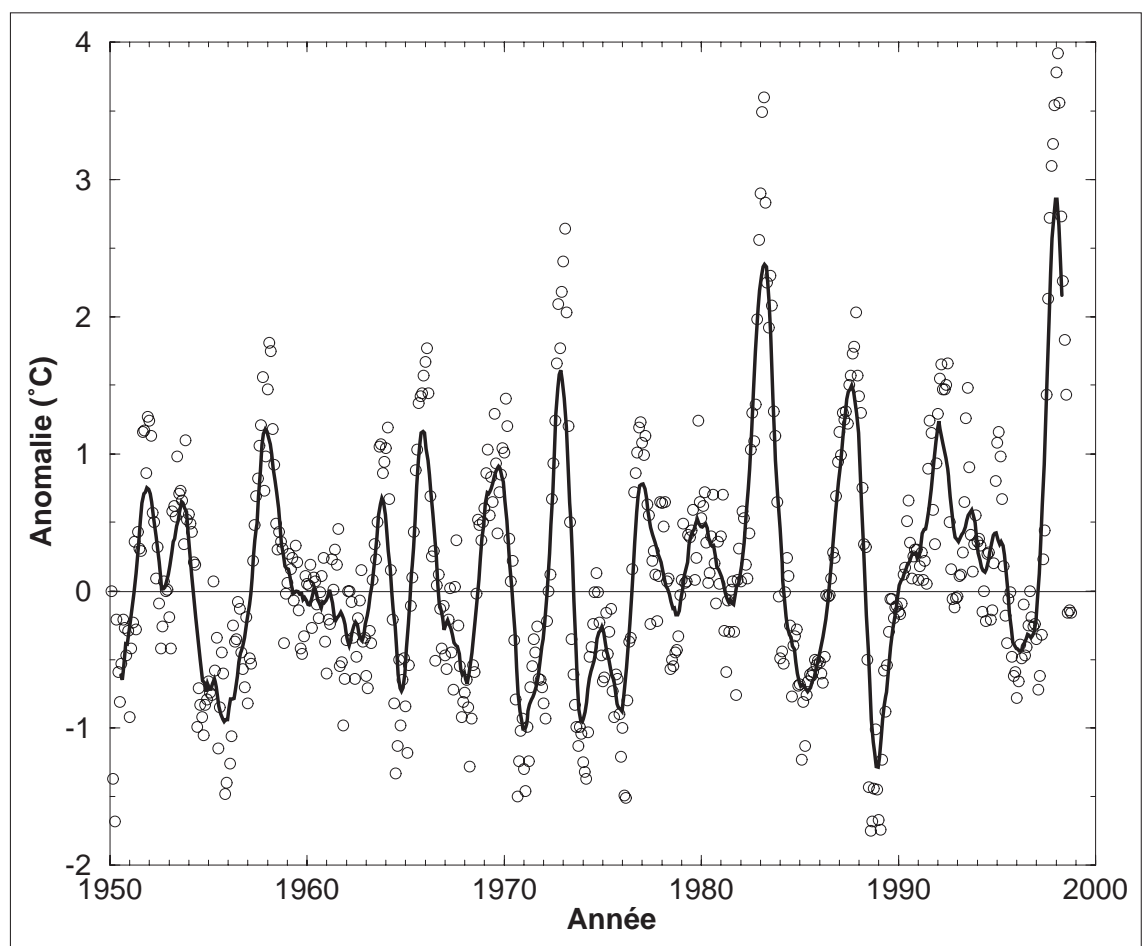

Figure 14 - Évolution observée depuis 1950 des anomalies de la température de surface de l'océan dans le Pacifique équatorial (région Niño-3 : $5^{\circ} \mathrm{S}-5^{\circ} \mathrm{N} ; 150^{\circ} \mathrm{W}-90^{\circ} \mathrm{W}$ ). Les cercles représentent les valeurs mensuelles, la courbe en trait plein une moyenne mobile sur un an. Les fortes anomalies positives correspondent aux événements chauds El Niño. (Source des données : Climate Prediction Center, NOAA, US Department of Commerce, http://nic.fb4.noaa.gov/data/cddb/ ) 
scepticisme concernant l'augmentation de l'effet de serre, évoque la possibilité de rétroactions liées à une possible augmentation des émissions de DMS par la biosphère marine, en réponse au réchauffement de l'océan superficiel, qui conduirait à une augmentation des noyaux de condensation pouvant modifier les propriétés radiatives des nuages. Bien que de tels mécanismes apparaissent plausibles, ils n'ont pas encore fait l'objet d'études quantitatives suffisantes pour que l'on puisse apprécier leur ordre de grandeur, et demeurent donc hypothétiques. En l'état actuel des connaissances, l'estimation de l'intensité du réchauffement global et de ses effets reste encore relativement imprécise, mais rien ne justifie une remise en cause du signe du changement climatique attendu.

\section{Remerciements}

BIBLIOGRAPHIE
Nous tenons à remercier Anny Cazenave, Pierre Barthelet et le Hadley Center pour nous avoir fourni des résultats non encore publiés.
Allen L. H. Jr., J. T. Baker et K. J. Boote, 1996 : The $\mathrm{CO}_{2}$ fertilization effect: higher carbohydrate production and retention as biomass and seed yield, in : Bazzaz F. et Sombroek W. (éds). Global Climate Change and Agricultural Production. John Wiley \& Sons, New York, 65-100.

Barthelet P., S. Bony, P. Braconnot, A. Braun, D. Cariolle, E. Cohensolal, J.-L. Dufresne, P. Delecluse, M. Déqué, L. Fairhead, M. A. Filiberti, M. Forichon, J.-Y. Grandpeix, E. Guilyardi, M. N. Houssais, M. Imbard, H. Le Treut, C. Levy, Z. X. Li, G. Madec, P. Marquet, O. Marti, S. Planton, L. Terray, O. Thual et S. Valcke, 1998 : Global coupled simulations of climate change due to increased atmospheric $\mathrm{CO}_{2}$ concentration. $C . R$. Acad. Sci. Paris, 326, série IIa, 677-684.

Basist A. N. et M. Chelliah, 1997 : Comparison of tropospheric temperatures derived from the NCEP/NCAR reanalysis, NCEP operational analysis, and the Microwave Sounding Unit. Bull. Amer. Meteor. Soc., 78, 1431-1447.

Battisti D. S. et E. S. Sarachik, 1995 : Understanding and predicting Enso. Rev. Geophys., $33,1367-1376$.

Becker M., G. D. Bert, J. Bouchon, J.-L. Dupouet, J.-F. Picard et E. Ulrich, 1995 : Longterm changes in forest productivity in northeastern France: The dendroecological approach. Landmann G. et M. Bonneau (éds). Forest Decline and Atmospheric Deposition Effects in the French Mountains. Springer-Verlag, Berlin, Heidelberg, New York, 143-156.

Berger A., 1988 : Milankovitch theory and climate. Rev. Geophys., 26, 624-657.

Carnell R. E. et C. A. Senior, 1998 : Changes in mid latitude variability due to increasing greenhouse gases and sulphate aerosols. Climate Dyn., 14, 369-383.

Casella E., J.-F. Soussana et P. Loiseau, 1996 : Long-term effects of $\mathrm{CO}_{2}$ enrichment and temperature increase on a temperate grass sward - I. Productivity and water use. Plant and Soil, 182, 83-99.

Cazenave A., K. Dominh, M. C. Gennero et B. Ferret, 1998 : Global mean sea level changes observed by Topex-Poseidon and ERS-1. Phys. Chem. Earth, 23, 9-10, 1069-1075.

Chahine M. T., 1992 : The hydrological cycle and its influence on climate. Nature, 359, 373-380.

Chahine M. T., R. Haskins et E. Fetzer, 1997 : Observation of the recycling rate of moisture in the atmosphere: 1988-1994. GEWEX News (WRCP), 7, 4, 1-4.

Cure J. D. et B. Acock, 1986 : Crop responses to carbon dioxide doubling: A literature survey. Agr. Forest Meteor., 38, 127-145.

Déqué M., P. Marquet et R. G. Jones, 1998 : Simulation of climate change over Europe using a global variable resolution general circulation model. Climate Dyn., 14, 173-189.

Gornitz V., 1995 : Monitoring sea level changes. Clim. Change, 31, 515-544.

Hansen J., M. Sato et R. Ruedy, 1997: Radiative forcing and climate response. J. Geophys. Res. - Atmos., 102, 6831-6864.

Hegerl G. C., H. von Storch, K. Hasselmann, B. D. Santer, U. Cubasch et P. D. Jones, 1996 : Detecting greenhouse-gas-induced climate change with an optimal fingerprint method. J. Climate, 9, 2281-2306. 
Houghton J. T., L. G. Meiro Filho, B. A. Callander, N. Harris, A. Kattenberg et K. Maskell (éds), 1996 : Climate Change 1995 - The Science of Climate Change, Cambridge University Press, New York, 572 p. (Contribution of WGI to the Second Assessment Report of the Intergovernmental Panel on Climate Change).

Hulme M., 1992 : A 1951-80 global land precipitation climatology for the evaluation of general circulation models. Climate Dyn., 7, 57-72.

Idso S. B., 1998 : $\mathrm{CO}_{2}$-induced global warming: a skeptic's view of potential climate change. Clim. Res., 10, 69-82.

Jones P. D., 1994 : Hemispheric surface air temperature variations: A reanalysis and an update to 1993. J. Climate, 7, 1794-1802.

Jones P. D., 1995 : Land surface temperatures: is the network good enough? Clim. Change, 31, 545-558.

Karl T. R. et R. W. Knight, 1998 : Secular trends of precipitation amount, frequency, and intensity in the United States. Bull. Amer. Meteor. Soc., 79, 231-241.

Kauppi P. E., K. Mielikäinen et K. Kuusela, 1992 : Biomass and carbon budget of European forests, 1971 to 1990. Science, 256, 70-74.

Keeling C. D., J. F. S. Chin et T. P. Whorf, 1996 : Increased activity of northern vegetation inferred from atmospheric $\mathrm{CO}_{2}$ measurements. Nature, 382, 146-149.

Knutson T. R., S. Manabe et D. F. Gu, 1997 : Simulated Enso in a global coupled ocean-atmosphere model: Multidecadal amplitude modulation and $\mathrm{CO}_{2}$ sensitivity. J. Climate, 10, 138-161.

Le Provost C., 1991 : Le niveau de la mer, un index fondamental pour l'océanographie et la climatologie. La Météorologie 7érie, 40, 3-12.

Lindzen R. S., 1990 : Some coolness concerning global warming. Bull. Amer. Meteor. Soc., 71, 288-299.

Manabe S. et R. J. Stouffer, 1997 : Climate variability of a coupled ocean-atmosphereland surface model: Implication for the detection of global warming (Walter Orr Roberts lecture). Bull. Amer. Meteor. Soc., 78, 1177-1185.

Meehl G. A. et W. M. Washington, 1996 : El Niño-like climate change in a model with increased atmospheric $\mathrm{CO}_{2}$ concentrations. Nature, 382, 56-60.

Mitchell J. F. B., R. A. Davis, W. J. Ingram et C. A. Senior, 1995 : On surface temperature, greenhouse gases, and aerosols: models and observations. J. Climate, 8, 2364-2386.

Mousseau M., E. Dufrêne, A. El Kohen, D. Epron, D. Godard, R. Liozon, J.-Y. Pontailler et B. Saugier, 1996 : Growth strategy and tree response to elevated $\mathrm{CO}_{2}$ : A comparison of beech (Fagus sylvatica L.) and sweet chestnut (Castanea sativa Mill.), in: Koch G. W. et H. A. Mooney (éds). Carbon Dioxide and Terrestrial Ecosystems, Academic Press, San Diego, États-Unis, 71-86.

Myneni R. B., C. D. Keeling, C. J. Tucker, G. Asrar et R. R. Nemani, 1997 : Increased plant growth in the northern high latitudes from 1981 to 1991. Nature, 386, 698-702.

Parker D. E., P. D. Jones, C. K. Folland et A. Bevan, 1994 : Interdecadal changes of surface temperature since the late 19th century. J. Geophys. Res. - Atmos., 99, 14373-14399.

Philander S. G., 1990 : El Niño, La Niña, and the Southern Oscillation. Academic Press, San Diego, États-Unis, 293 p.

Pirazzoli P. A., 1996 : Sea-Level Changes - The Last 20000 Years. John Wiley \& Sons, Chichester, Royaume-Uni, $210 \mathrm{p}$.

Reader M. C. et G. J. Boer, 1998 : The modification of greenhouse gas warming by the direct effect of sulphate aerosols. Climate Dyn., 14, 593-607.

Santer B. D., K. E. Taylor, T. M. L. Wigley, J. E. Penner, P. D. Jones et U. Cubasch, 1995 : Towards the detection and attribution of an anthropogenic effect on climate. Climate Dyn., 12, 77-100.

Saugier B., 1998 : Perspectives for future research, in: Jarvis P. G. (éd.). The Likely Impact of Rising $\mathrm{CO}_{2}$ and Temperature on European Forests, Cambridge University Press, Cambridge, 344-352.

Tett S. F. B., J. F. B. Mitchell, D. E. Parker et M. R. Allen, 1996 : Human influence on the atmospheric vertical temperature structure: Detection and observations. Science, 274, 1170-1173.

Trenberth K. E. et T. J. Hoar, 1997 : El Niño and climate change. Geophys. Res. Lett., 24, 3057-3060. 